\title{
Enhancing Design Features of Asymmetric Spur Gears Operating on a Specified Center Distance Using Tooth Sum Altered Gear Geometry
}

\author{
Avil Allwyn Dsa ${ }^{1, *}$, Joseph Gonsalvis ${ }^{2}$ \\ ${ }^{1}$ Department of Mechanical Engineering, Don Bosco College of Engineering, Goa, India \\ ${ }^{2}$ Department of Mechanical Engineering, RNS Institute of Technology, Bangalore, India \\ Received 28 January 2021; received in revised form 11 March 2021; accepted 12 March 2021 \\ DOI: https://doi.org/10.46604/peti.2021.7074
}

\begin{abstract}
Asymmetric gears have evolved from the rising demand for power transmission drives with high load-carrying capacity, surface durability, and service life. Direct design and $\mathrm{S} \pm$ profile shifted system are the most common approaches used for enhancing design features by geometry modification in asymmetric gears. This paper aims at establishing asymmetric gear geometry modification using tooth sum alteration for a family of gears running on a specified center distance as a feasible design approach. A complete mathematical treatment of the design approach is provided, and an in-house developed computer program is used for numerical simulation. The paper explores the influence of dynamic load factors, location factors for bending, specific sliding on load-bearing capacity, and surface durability on different tooth sum alterations. The study concludes that tooth sum altered asymmetric gear geometry can be employed as an effective design technique that offers designers flexibility in designing gears for surface wear, load-bearing, and tooth life.
\end{abstract}

Keywords: asymmetric gear, altered tooth sum, specific sliding, dynamic load, wear

\section{Introduction}

In recent years nonstandard asymmetric gear design has been researched for devising ways to enhance the performance of gear drives. The published research indicates attempts to improvise design features by varying the drive or coast side pressure angle for varying the tooth thickness and addendum height for contact ratio. These changes are brought about either by altering the cutter geometry or by profile shifting. The major limitation of these methods is the need to have custom-made tooling for the former and the need to trade off variation in the center distance for the latter. A method to alter the tooth geometry for enhanced design features without custom-made tooling or varying the center distance would indeed be novel.

Earlier, Kapelevich [1] proposed a method for the design of gears with asymmetric teeth. Several equations required for the design of asymmetric gears are developed for the synthesis of asymmetric gears. Litvin, Lian, and Kapelevich [2] proposed gear geometry modification of asymmetric gears for the reduction in transmission error by using a combination of involute gears and double crowned pinion with a larger drive side pressure angle. Karpat, Ekwaro-Osire, Cavdar, and Babalik [3] studied the dynamic characteristics of asymmetric spur gears by addendum modified gear geometry for the reduction in transmission error and dynamic factor. Karpat and Ekwaro-Osire [4] proposed tooth profile tip relief modification as an effective measure to reduce dynamic loads and wear. Sekar [5] made a comparative study of load sharing, fillet, and contact stresses in symmetric and asymmetric gear drives. Sekar and Muthuveerappan [6] estimated the tooth form factor and stress

* Corresponding author. E-mail address: avil.dsa@ dbcegoa.ac.in

Tel.: +91832-2777344; Mobile: +91-9657043438 
correction factor for asymmetric gears using standard ISO-B methodology. Gonsalvis and Rayudu [7] reported the sensitivity of the contact ratio to tooth-sum alteration in symmetric gears, its influence on operating pressure angle, and profile shift coefficient. Avil and Gonsalvis [8] developed a methodology to evaluate dynamic load distribution in tooth-sum altered symmetric gears considering the altering root fillet geometry. Gonsalvis and Sachidananda [9] explored the possibility of altering the tooth-sum of symmetric gear-pair, to tailor the gear drive either for low, normal, or high contact ratio.

While literature related to tooth sum altered symmetric gear geometry is scarce, however, the published literature reveals no reference on the benefits and problems of accommodating tooth sum altered profile shifted asymmetric gears running on a specified center distance. The present work attempts to explore the possibility of modifying the tooth geometry of an asymmetric spur gear-pair for design benefits by altering the tooth sum while holding the operating center distance constant, which also can be named as Tooth-Sum Altered Asymmetric Gear (TSAAG). The objective of this work is to establish mathematical expressions for the TSAAG geometry, check for kinematic compatibility and design superiority of TSAAG over normal contact ratio (NCR) asymmetric counterparts. A case study on altering the tooth sum of an asymmetric spur gear of a given module to obtain possible TSAAG combinations is modeled as a single degree of freedom spring-mass-damper system (SMD) [10-11] subjected to forced vibration, for evaluating the dynamic load factors, load-bearing ability and specific sliding characteristics with NCR asymmetric counterpart.

\section{The Geometry of TSAAG}

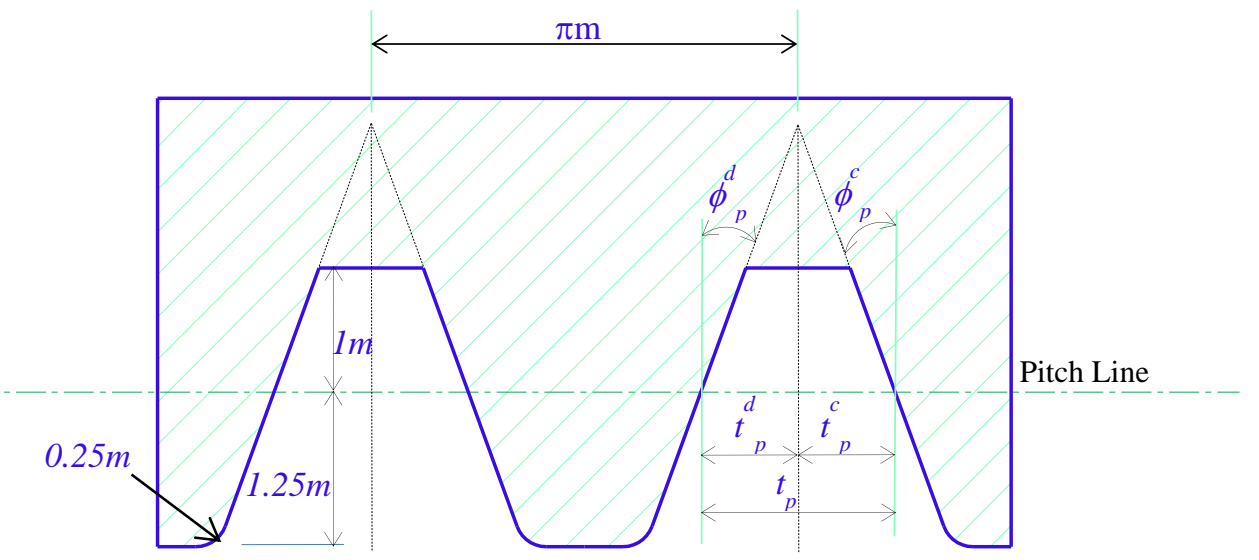

Fig. 1 Tooth thickness distribution factor

An asymmetric rack cutter of module $m$, with standard proportions, is shown for reference in Fig. 1. The drive and the coast side pressure angles are denoted by $\phi_{p}^{d}$ and $\phi_{p}^{c}$ respectively. For convenience, the authors define drive side tooth thickness $t_{p}^{d}$, as a factor $\tau$ of total tooth thickness $t_{p}$, along the pitch line. From the geometry of the rack we get

$$
\begin{aligned}
& t_{p}^{d}=\tau t_{p} \\
& \frac{\tan \phi_{p}^{c}}{\tan \phi_{p}^{d}}=\frac{1-\tau}{\tau}
\end{aligned}
$$

The general form of expression for tooth thickness $t_{x}[1]$ at any arbitrary location for profile shifted asymmetric spur gear of module $m$ with total profile shift coefficient $X_{S}[12]$ and shift factor $\kappa$ is given by

$$
t_{x}=r_{x}\left(\frac{t_{p}}{r_{p}}+\Lambda_{x}\right)
$$




$$
\begin{aligned}
& t_{p}=\frac{P_{c}}{2}+\kappa X_{s} m\left(\tan \phi_{p}^{d}+\tan \phi_{p}^{c}\right) \\
& \Lambda_{x}=\left[\left(\operatorname{inv} \phi_{p}-i n v \phi_{x}\right)^{d}+\left(\operatorname{inv} \phi_{p}-i n v \phi_{x}\right)^{c}\right]
\end{aligned}
$$

\subsection{Total profile shift in TSAAG system}

The need for profile shifting in TSAAG arises due to tooth sum alteration and the constraint of constant center distance. The geometrical consequences of profile shifting in a TSAAG system is either an increase or decrease in the tooth thickness, restoring physical contact between the teeth, and also an increase or decrease in the radius of the addendum circle maintaining the overall tooth height. For continual conjugate action, a calculated total profile shift coefficient $X_{S}$ is divided among the meshing gears. Mathematically total profile shift coefficient $X_{s}$, for a TSAAG system with design backlash B, operating on a working pressure angle $\phi_{w}^{d}$ and radius $r_{w}$ with tooth thickness $t_{w}$ on the driver and driven is given as

$$
t_{w 1}+t_{w 2}+B=\frac{2 \pi r_{w 1}}{z_{1}}
$$

Applying Eq. (3) for tooth thickness at working pitch circle, Eq. (6) can be written as

$$
r_{w 1}\left(\frac{t_{p 1}}{r_{p 1}}+\Lambda_{w}\right)+r_{w 2}\left(\frac{t_{p 2}}{r_{p 2}}+\Lambda_{w}\right)+B=\frac{2 \pi r_{w 1}}{z_{1}}
$$

For any profile shifted system the ratio of tooth numbers is equal to the ratio of the working pitch circle radii

$$
\frac{r_{w 2}}{r_{w 1}}=\left(\frac{z_{2}}{z_{1}}\right)^{a}
$$

Substituting Eq. (8) in Eq.(7), replacing $\left(z_{1}^{a}+z_{2}^{a}\right)=Z_{s}^{a}$ and dividing throughout by $\left(2 r_{w 1}\right)$, Eq. (7) can we rearranged as

$$
t_{p 1}+t_{p 2}=m\left(\pi-\frac{1}{2} \Lambda_{w} Z_{s}^{a}-\frac{B z_{1}}{2 r_{w 1}}\right)
$$

If the profile shift coefficient of the pinion is $x_{1}=\left(\kappa X_{S}\right)$, then the shift coefficient of the gear is $x_{2}=\left(1-\kappa X_{s}\right)$. Using Eq. (4), Eq. (9) is written as

$$
m\left[\pi+X_{s}\left(\tan \phi_{p}^{d}+\tan \phi_{p}^{c}\right)\right]=m\left(\pi-\frac{1}{2} \Lambda_{w} Z_{s}^{a}-\frac{B z_{1}}{2 r_{w 1}}\right)
$$

Rearranging Eq. (10), the general form of the expression for total profile shift coefficient can be expressed as

$$
X_{s}=\frac{-m \Lambda_{w} \beta Z_{s}^{a}-2 \alpha B}{2 m \beta\left(\tan \phi_{p}^{d}+\tan \phi_{p}^{c}\right)}
$$

The value of the total shift coefficient is constant for a TSAAG gear pair as long as the sum of the drive side and coast side pressure angles is unchanged. The coordinates of the drive and coast-side involute for profile shifted asymmetric gear are given by 


$$
\begin{aligned}
& x=r_{x} \cos \psi_{x}, y=r_{x} \sin \psi_{x} \\
& \psi_{x}=\frac{\pi}{2}+\frac{\tau}{z}\left[\pi+2 \kappa X_{s}\left(\tan \phi_{p}^{d}+\tan \phi_{p}^{c}\right)\right]+\left(\operatorname{inv} \phi_{p}^{d}-\operatorname{inv} \phi_{x}^{d}\right)
\end{aligned}
$$

\subsection{Pressure angles in TSAAG system}

An asymmetric gear pair with reference tooth-sum $Z_{s}^{r}$ and drive side pressure angle $\phi_{p}^{d r}$, operating on a center distance $c_{S}^{r}$ can be replaced by a tooth-sum altered $Z_{s}^{a}$ asymmetric gear-pair, operating at working pressure angle $\phi_{w}^{d a}$, keeping the center distance unchanged $c_{w}^{d}=c_{s}^{r}$ (TSAAG system). If the tooth sum is altered by a factor $\alpha$, then

$$
Z_{s}^{a}=\alpha Z_{s}^{r}
$$

The relation between the number of teeth and radius of the working pitch circle is given by

$$
z=\frac{2 r_{w}}{m} \cdot \frac{\cos \phi_{w}^{d}}{\cos \phi_{p}^{d}}
$$

Using the Eq. (15), Eq. (14) can be reframed as

$$
\left(\frac{\cos \phi_{w}^{d}}{\cos \phi_{p}^{d}}\right)^{a}\left(r_{w 1}+r_{w 2}\right)^{a}=\alpha\left(r_{1}+r_{2}\right)^{r}
$$

Using the relation $\left(r_{w 1}+r_{w 2}\right)=c_{w},\left(r_{1}+r_{2}\right)=c_{s}$ and defining $c_{w}^{a}=\beta c_{s}^{r}$, Eq. (16) can be rearranged to express the operating pressure angles on the drive and the coast side.

$$
\left\{\begin{array}{l}
\phi_{w}^{d}=\cos ^{-1}\left(\frac{\alpha}{\beta} \times \cos \phi_{p}^{d}\right) \\
\phi_{w}^{c}=\cos ^{-1}\left(\frac{\alpha}{\beta} \times \cos \phi_{p}^{c}\right)
\end{array}\right.
$$

Reframing Eq. (16) to relate the working center distance of TSAAG with its tooth sum we get

$$
c_{w}^{a}=\left(\frac{\cos \phi_{p}^{d}}{\cos \phi_{w}^{d}}\right)^{a} \times\left(\frac{m}{2}\right)^{r} \times Z_{s}^{a}
$$

The standard center distance of the TSAAG is related to its tooth sum by the relation

$$
c_{s}^{a}=\left(\frac{m}{2}\right)^{a} \times Z_{s}^{a}
$$

Subtracting Eq. (19) from Eq. (18), assuming that the TSAAG module remains unchanged

$$
\left(c_{w}-c_{s}\right)^{a}=\left(\frac{m}{2}\right)^{r} \times Z_{s}^{a}\left(\frac{\beta}{\alpha}-1\right)
$$

\subsection{Topping for TSAAG system}

The TSAAG is a profile shifted system on fixed center distance. For a given profile shift, the ratio of addendum radius gain to that of tooth thickness gain along the pressure line is always constant and is approximately equal to 3 (refer to Fig. 2). This significant increase in addendum radius for attaining a nominal increase in tooth thickness along the pressure line may 
cause inappropriate root clearance. Similarly, a small amount of negative shift results in a considerable reduction in the magnitude of the addendum circle radius. This issue can be overcome by topping the addendum with a calculated positive or negative value. To ensure appropriate root clearance, addendum radii are topped with tooth topping coefficient Y. A TSAAG generated by a rack cutter with an addendum of 1.25 module and dedendum of 1 module in mesh is shown in Fig. 3 .

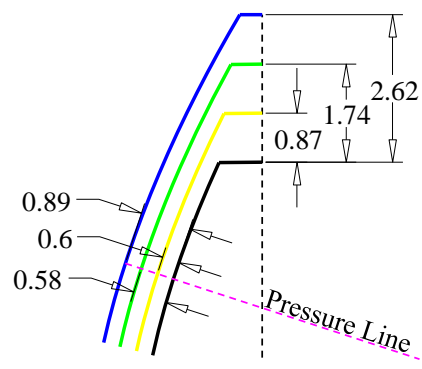

Fig. 2 Profile shift

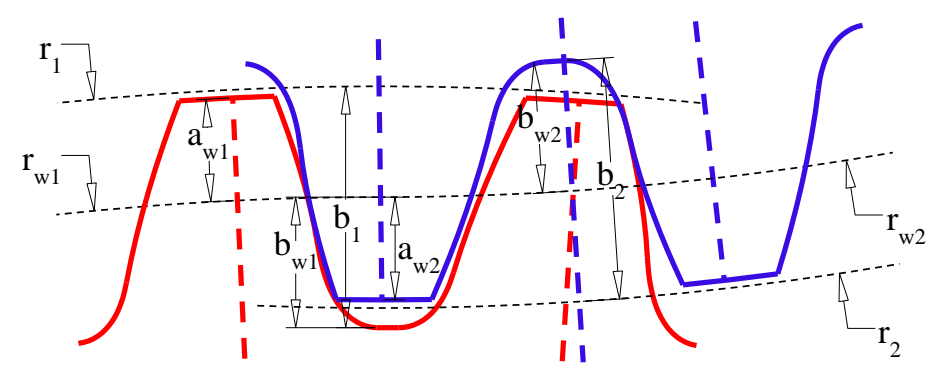

Fig. 3 Topping for root clearance

The radial root clearance $r_{c l}$ is expressed as

$$
r_{c l}=\left(b_{1}+b_{2}-2.25 m\right)-\left(r_{1}+r_{2}\right)+\left(r_{w 1}+r_{w 2}\right)
$$

The radial distance between the root circle and the pitch circle is given by

$$
\left\{\begin{array}{l}
b_{1}=\left(1.25-\kappa X_{s}\right) m \\
b_{2}=\left[1.25-\left(1-\kappa X_{s}\right) m\right]
\end{array}\right.
$$

Using Eq. (22), Eq. (21) can be reframed as

$$
X_{s}=\frac{\left(0.25 m-r_{c l}\right)+\left(c_{w}-c_{s}\right)^{a}}{m}
$$

Using Eq. (20) in Eq. (23), and defining $\left(0.25 m-r_{c l}\right)=Y m$, an expression for tooth topping coefficient is given by

$$
Y=X_{s}+\frac{Z_{s}^{a}}{2}\left(1-\frac{\beta}{\alpha}\right)
$$

The root clearance for any gear pair is established to be at least $0.25 \mathrm{~m}$. TSAAG system which is topped should satisfy the expression

$$
r_{c l}+Y m=0.25 m
$$

TSAAG system works on the concept of altering the tooth sum of the reference gear pair. The parameters $\alpha$ and $\beta$ enables designers to opt for tooth sum altered TSAAG system or center distance altered S \pm asymmetric profile shifted system. The Eqs. (1)-(25) are the most general set of expressions for TSAAG profile shifted system, $\mathrm{S} \pm$ profile shifted system, and standard spur 
gear system (STS). For a standard backlash-free gear-pair, $\alpha=\beta=1$. For the TSAAG system, $0.96<\alpha<1.04$ and $\beta=1$. For the $\mathrm{S} \pm$ profile shifted system, $0.96<\beta<1.04$ and $\alpha=1$. TSAAG accommodates sums of various curvatures by keeping the center distance constant and changing operating pressure angles, whereas the $\mathrm{S} \pm$ asymmetric profile shifted system accommodates sums of various curvatures by keeping the sum of the base circle radii constant and changing operating pressure angles (refer to Fig. 4). Any two arbitrary gear pairs operate at the same working pressure angles as long as the ratio $(\alpha / \beta)$ is maintained constant. The magnitude of the total profile shift coefficient remains constant for any asymmetric gear pair as long as the sum of the drive side and coast side pressure angles are unchanged, referring to Eq. (11).

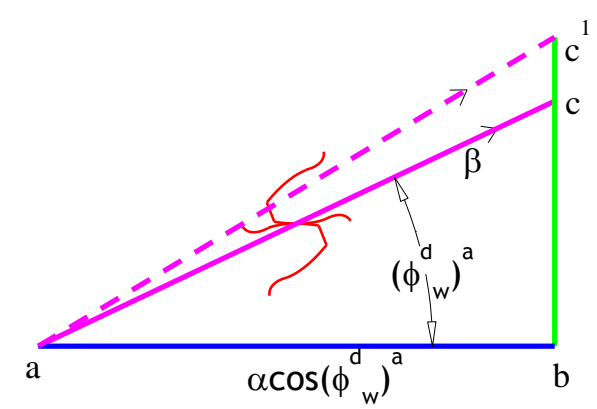

(a) Center distance altered gears

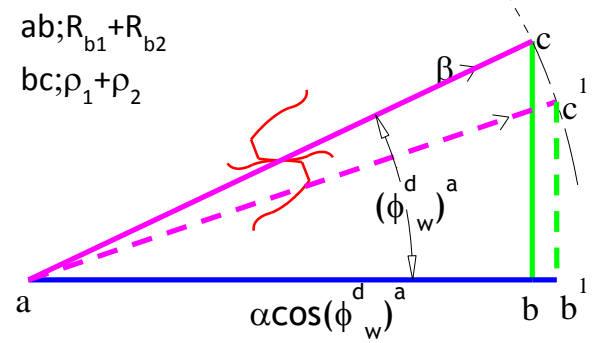

(b) Tooth sum altered gears

Fig. 4 Difference between TSAAG and S6 approach

\subsection{Contact ratio and interference avoidance criteria for TSAAG}

It is possible to accommodate gear pairs with different modules and tooth sums on a specified center distance. Operating higher module gears with lower tooth sums increases power transmission capability but reduces contact ratio and restricts speed range. Gear ratios achievable with any tooth-sum should satisfy the interference avoidance criterion [13] which also establishes the limiting value for the module. The necessary and sufficient mathematical conditions are given as

$$
\begin{aligned}
& \theta_{r a} \leq \theta_{\max } \\
& r_{f} \leq r_{l}
\end{aligned}
$$

and

$$
C R=\frac{z_{1}}{2 \pi}\left(\theta_{r a}-\theta_{r l}\right)
$$

\section{Safe Load Transmission Capability of TSAAG System}

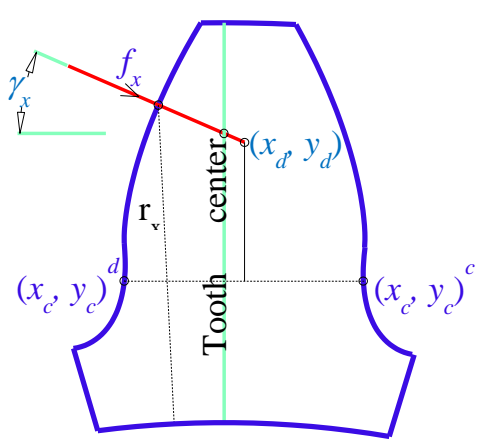

(a) Tooth subjected to a load

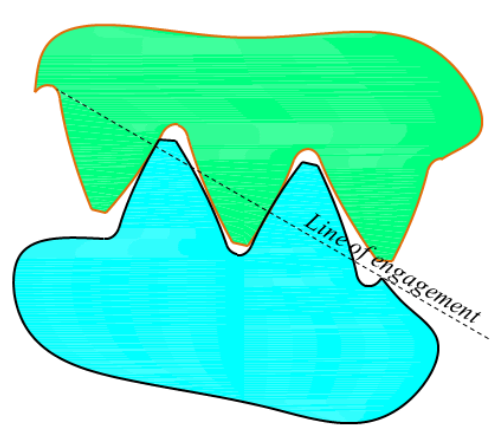

(b) A gear pair in mesh

Fig. 5 Load bearing in a TSAAG system

Evaluation of power transmission capability based on material's bending strength depends upon the tooth geometry. A tooth load intensity $f_{x}$ per unit face width along the pressure line at any arbitrary value of roll angle can be resolved into components along and perpendicular to the tooth centerline. The tangential component of the tooth force along the pressure 
line causes tensile stresses on one side of the root fillet and compressive stress on the other side. The effect of the radial component of the tooth force is to cause compressive stress on either side of the tooth. The net effective fillet stress at the root is compressive on one side and tensile on the other. These cyclic tensile stresses are responsible for the initiation and propagation of fatigue cracks. The expression for safe load-bearing $F$ [13] is modified to find the maximum static load which could be safely transmitted by a gear of a given module and material to avoid bending failure in any kind of spur gear system (refer to Fig. 5).

$$
\begin{aligned}
& F=\frac{\sigma_{f}}{k_{t} x_{d l}} m \\
& k_{t}=m k_{f} \cos \gamma_{x}\left[\frac{\left(y_{d}-y_{c}^{m}\right)}{Z}-\frac{\tan \gamma_{x}}{A}\right] \\
& x_{s l}=\frac{\lambda_{x}}{\lambda_{e q}}
\end{aligned}
$$

where $k_{t}$ is called the geometric location factor for bending, $A$ and $Z$ are the cross-sectional area and the sectional modulus of the critical section. The Cartesian coordinates of the critical section on the drive side and the coast side are $\left(x_{c}^{d}, y_{c}^{d}\right)$ and $\left(x_{c}^{c}, y_{c}^{c}\right)$ respectively. Dynamic load factor $x_{d l}$ is the ratio of location-based mesh stiffness $\lambda_{x}$ to total mesh stiffness $\lambda_{e q}$.

\section{Surface Wear in TSAAG System}

Gear surface wear is an on-going process that results in loss of material along the tooth profile with an increasing number of load cycles. The profile deviation increases as wear progresses, the effects of which are manifested in the form of an increase in noise, vibration levels, and backlash. These deviations generate overloads on the teeth for which the gearing may not have been sufficiently dimensioned when designed, which could cause the onset of the other modes of failure in the spur gear tooth. Surface wear is evaluated by the volume of material lost, and the degree of wear is described by wear rate, specific wear rate, or wear coefficient. Archards wear equation [14] for sliding contacts is a mechanical wear model popular for its simplicity and its ability to characterize wear under a wide variety of conditions. Flodin and Anderson [15] modified the generalized Archard's equation to describe dry and boundary lubricated sliding surfaces of a gear pair in mesh.

$$
h_{x, n}=h_{x, n-1}+2 k_{w} \sigma_{f c} a_{0} \frac{v_{r 1}-v_{r 2}}{v_{r 1}}
$$

Observing term within the parenthesis $\left[v_{r 1}-v_{r 2} / v_{r 1}\right]=V_{s S}$ called as specific sliding, and $\sigma_{f c} \alpha_{0}=2 f_{d x} / \pi$ [12], Eq. (31) can be modified as

$$
\left\{\begin{array}{l}
h_{x, n}=h_{x, n-1}+4 k_{w} \frac{f_{d x}}{\pi} V_{s s} \\
h_{x, n}=h_{x, n-1}+4 x_{d l} V_{s s} \frac{F}{\pi} * k_{w}
\end{array}\right.
$$

where $F$ is the static load transmitted per unit face width, $h_{x n}$ is the accumulated wear at any location after $n$ cycles, $f_{d x}$ is the dynamic load per unit face width, $x_{d l}$ is the dynamic load factor at the contact location, $\alpha_{0}$ is the semi-contact width of the elliptical contact, and $k_{w}$ is the wear coefficient. The total profile error $\varepsilon_{x}$, dynamic load $f_{d x}$, and load factor $x_{d l}$ at any location after $n$ cycles is given by 


$$
\begin{aligned}
& \varepsilon_{x n}=h_{x n}^{1}+h_{x n}^{2} \\
& f_{d x}=\lambda_{x}\left(x_{r}-\varepsilon_{x}\right) \\
& x_{d l}=\frac{f_{d x}}{F}
\end{aligned}
$$

\section{Implementation Scheme and Methodology}

For the case study, a gear pair of module 2 with a drive side pressure angle $20^{\circ}$ and tooth thickness distribution factor $\tau=0.438$ is adopted for a center distance of $108 \mathrm{~mm}$. Operating conditions created by four different tooth-sum alteration factors $0.96<\alpha<1.04$ and profile shift factors with $\beta=1$ are considered. The material properties, operating conditions, and gear geometry alterations described by Eqs. (11), (17), and (24), are tabulated in Table 1.

Table 1 TSAAG geometry and material properties

\begin{tabular}{|c|c|c|c|c|c|c|c|}
\hline $\mathrm{GR}=1: 1$ & \multicolumn{5}{|c|}{$\alpha$} & \multicolumn{2}{c|}{ Material properties and operating conditions } \\
\hline$\alpha$ & 0.96296 & 0.98148 & 1 & 1.01852 & $1.03703 *$ & Density of steel & $7700 \mathrm{~kg} / \mathrm{m}^{3}$ \\
\hline$Z_{s}^{a}$ & 104 & 106 & 108 & 110 & 112 & Damping factor $(\xi)$ & 0.17 \\
\hline$\phi_{w}^{a}$ & 0.4397 & 0.3968 & 0.349 & 0.294 & 0.2264 & Young's modulus $(E)$ & $206 \mathrm{GPa}$ \\
\hline $\mathrm{B}$ & 0.144 & 0.144 & 0.144 & 0.144 & 0.144 & Fillet strength $\left(\sigma_{f}\right)$ & $413 \mathrm{MPa}$ for $10^{6} \mathrm{cycles}$ \\
\hline$X_{S}$ & 2.163 & 0.97 & -0.1 & -1.0273 & -1.7885 & Wear coefficient $\left(k_{w}\right)$ & $5 \times 10^{-16} \mathrm{~m}^{2} \mathrm{~N}^{-1}$ \\
\hline$Y$ & 0.1627 & -0.0302 & -0.1 & -0.0272 & 0.2111 & Speed & $1000 \mathrm{rpm}$ \\
\hline$t_{p}$ & 4.715 & 3.847 & 3.069 & 2.392 & 1.839 & *ase of interference \\
\hline
\end{tabular}

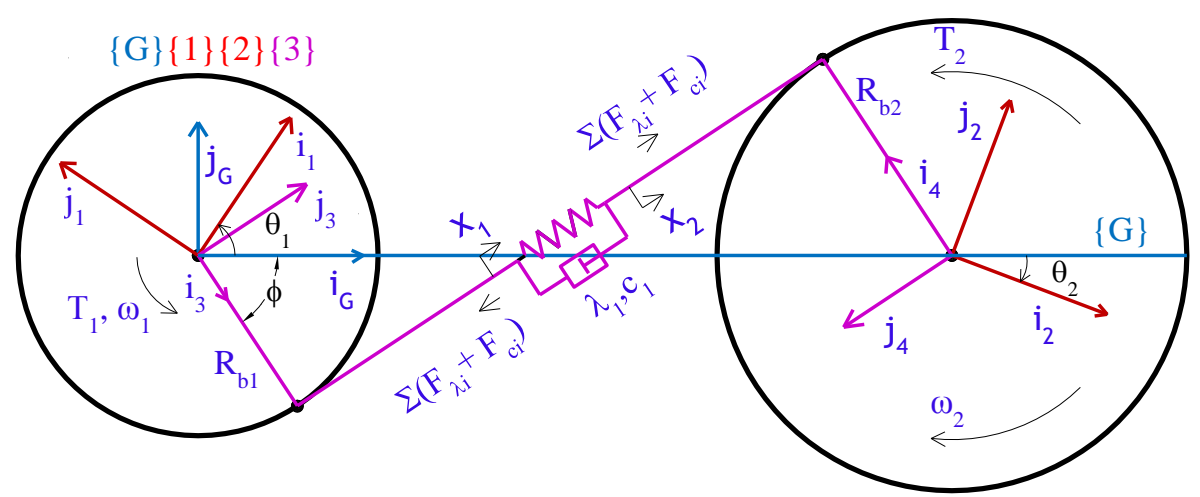

Fig. 6 Dynamic model for spur gear

The product of dynamic load factor and location-based geometric factor determines the safe load-bearing ability of a gear, referring to Eq. (28). The product of dynamic load factor and specific sliding is the deciding factor for accumulated wear depths over $n$ cycles, referring to Eq. (32). Hence the determination of dynamic load factors is critical in the analysis of any gearing system. To evaluate dynamic load factors for TSAAG, a gear pair in mesh is modeled as two rigid disks connected by a spring-damper set along the pressure line (refer to Fig. 6). The single Degree of Freedom (DOF) dynamic model considers the influence of mesh stiffness, damping forces $F_{c}$, friction forces, static transmission error at the mesh interface, and are expressed as a time-varying function. The gear pairs are assumed to be of unit face width and free of tooth profile errors. The differential equations of motion (EOM) for a single DOF spring mass damper system can be expressed as

$$
\begin{aligned}
& m_{e q} \ddot{x}_{r}+c_{e q} \dot{x}_{r}+k_{e q} x_{r}=F \\
& m_{e q}=\frac{m_{e 1} m_{e 2}}{m_{e 1}+m_{e 2}}
\end{aligned}
$$




$$
\begin{aligned}
& k_{e q}=\frac{m_{e 2} \sum_{i=1}^{n} \lambda_{i}\left(1 \pm \mu \theta_{1 i}\right)+m_{e 1} \sum_{i=1}^{n} \lambda_{i}\left(1 \pm \mu \theta_{2 i}\right)}{m_{e 1}+m_{e 2}} \\
& c_{e q}=2 \xi \sqrt{m_{e q} k_{e q}}
\end{aligned}
$$

where $m_{e q}, k_{e q}, c_{e q}$ are mass, stiffness, and damping coefficient of the equivalent S-M-D system, $m_{e}$ is the effective mass, and $x_{r}$ is the relative displacement at the mesh interface.

FEM analysis is adopted to create gear tooth stiffness $k_{s}$, for TSAAG system as a function of its roll angle. The coefficients for the polynomials for various $\alpha$ values are tabulated (refer to Fig. 7 and Table 2).

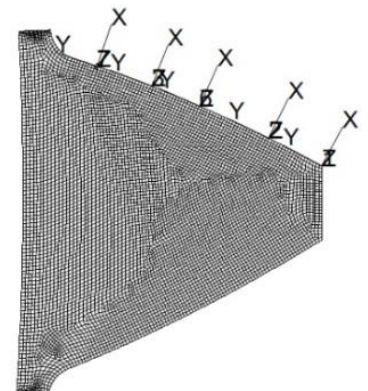

(a) $\alpha=0.96296, \kappa=0.5, \beta=1$

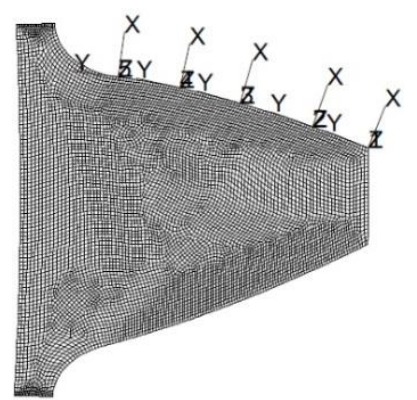

(d) $\alpha=1.01852, \kappa=0.5, \beta=1$

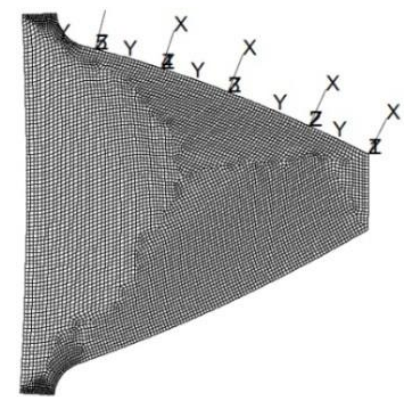

(b) $\alpha=0.98148, \kappa=0.5, \beta=1$

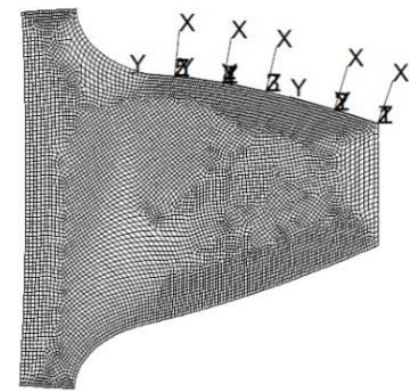

(e) $\alpha=1.03703, \kappa=0.5, \beta=1$

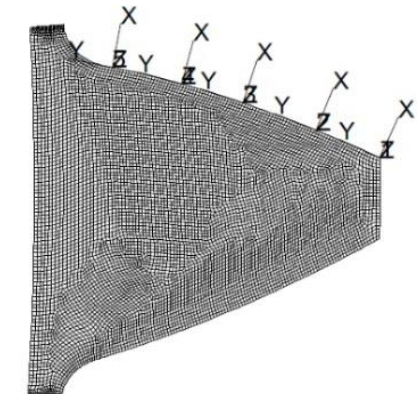

(c) $\alpha=1, \kappa=0.5, \beta=1$

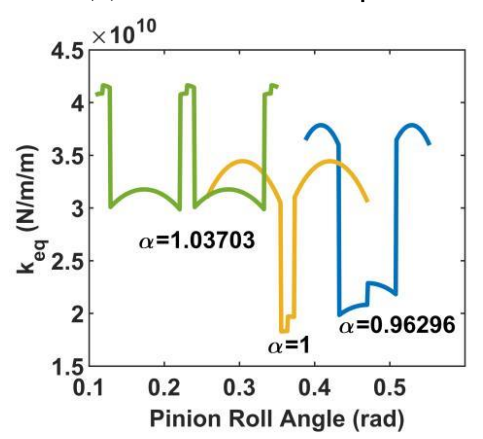

(f) Mesh stiffness of TSAAG

Fig. 7 FEM analysis for the stiffness of TSAAG

Table 2 Equation for stiffness of TSAAG

\begin{tabular}{|c|c|c|c|c|}
\hline \multicolumn{5}{|c|}{$k_{S}(\theta)=p_{1} \theta^{3}+p_{2} \theta^{2}+p_{3} \theta+p_{4}$} \\
\hline$\alpha$ & $\mathrm{p} 1$ & $\mathrm{p} 2$ & $\mathrm{p} 3$ & $\mathrm{p} 4$ \\
\hline 0.96296 & -7304.8 & 9601.2 & -4348.9 & 725.24 \\
\hline 0.98148 & -3595.8 & 4013.4 & -1631.5 & 284.15 \\
\hline 1 & -1780.7 & 1580.5 & -597.23 & 131.89 \\
\hline 1.01852 & -937.43 & 536.73 & -204.14 & 74.484 \\
\hline 1.03703 & -1049.9 & 427.48 & -133.25 & 54.627 \\
\hline
\end{tabular}

The equation of motion is numerically solved using the fourth-order Runge-Kutta linear iterative procedure. One mesh period is divided into a certain number of intervals and the solution procedure is started with a trial value of initial conditions. The values of the relative displacement and velocity after one mesh period are compared with the trail values. The solution is said to converge when the difference between the initial condition and the obtained values after one mesh cycle are within the preset tolerance. The value of relative displacement is used for finding dynamic load factors, referring to Eq. (35). The safe static load transmission capability is evaluated using the ratio of location-based mesh stiffness to total mesh stiffness. A flow chart of the in-house developed code is provided in Fig. 8. 


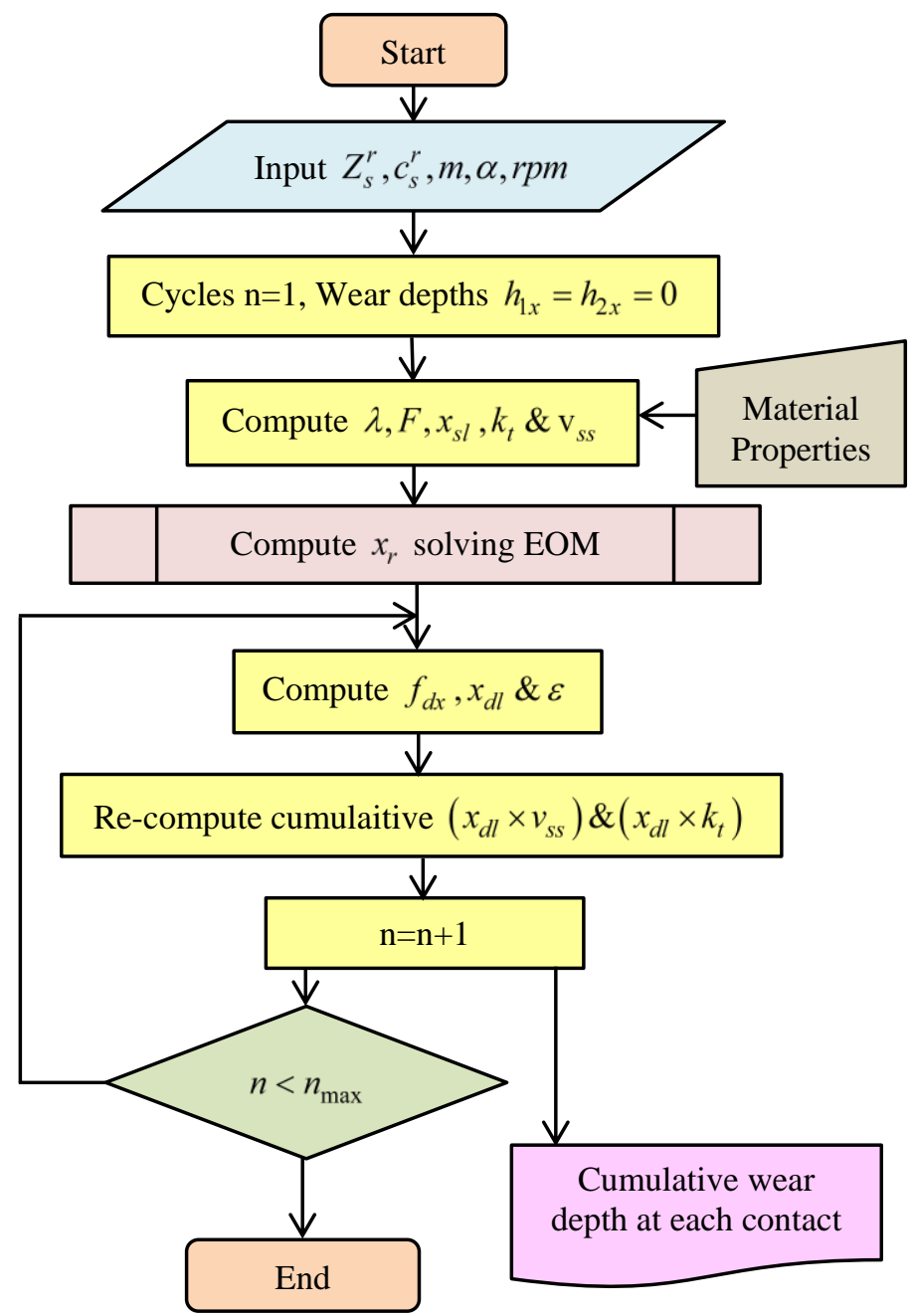

Fig. 8 Flow chart for evaluation of $\left(x_{d l} \times v_{s S}\right) \&\left(x_{d l} \times k_{t}\right)$

\section{Results and Discussion}

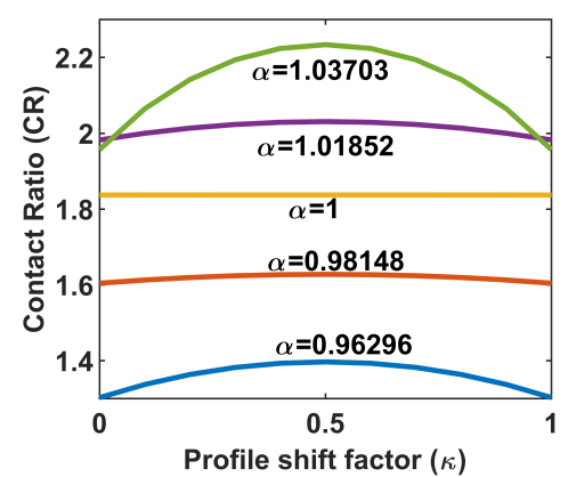

Fig. 9 Contact ratio vs. profile shift factor

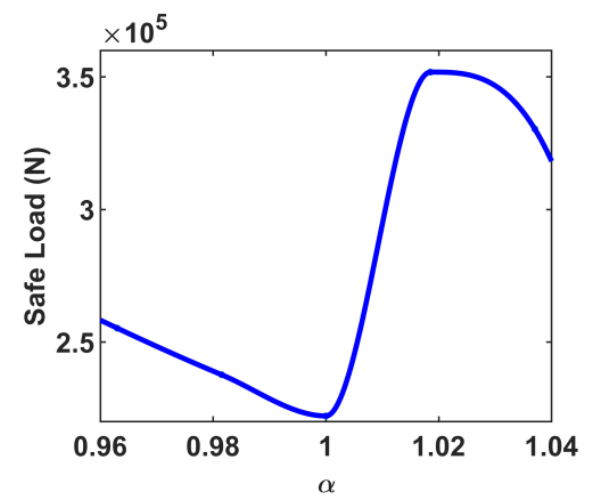

Fig. 10 Safe load vs. tooth sum alteration factor $(\alpha)$

Contact ratio is a geometric parameter of much importance which is an indicator of the influence of gear geometry on its performance. Since it is a pure geometric parameter, it is always a good starting point to begin an investigation that involves gear geometry modification. The investigation of the contact ratio provides information and direction in which the analysis needs to proceed. TSAAG is a tooth sum altered, profile shifted, addendum topped system, and all of these modifications affect the contact ratio. A TSAAG carrying positive profile shift exhibits a reduction in the contact ratio, owing to reduced tooth-sum, increased working pressure angle, and reduced radius of the addendum circle due to tooth topping. Limiting the study to gears with a contact ratio above 1.3 (refer to Fig. 9), it is observed that while the tooth-sum reduction by 4 is permissible with contact ratio falling to 1.36 , a tooth-sum increase by 2 is permissible, without causing interference, raising the contact ratio above 2 . It 
is important to note that +4 tooth alteration without any design backlash results in undercutting of gears (refer to Table 1) and would cause a certain part of the involute profile not to participate in load-bearing. This part of the involute profile would show an increase in the contact ratio mathematically but has no practical significance. Hence positive tooth alterations are advised only up to a profile shift value at which the first sign of undercutting appears. Similarly, the negative number of tooth alterations should be limited to a profile shift value at which the tooth becomes peaked. Attempting higher negative alterations would result in an unreasonable reduction in contact ratio. Introduction of design backlash reclaims profile shift to a certain degree, salvaging certain part of the addendum which would otherwise be topped. Hence one can expect negative number of tooth alterations without backlash to lower the contact ratio, but introduction of backlash shows an improvement of the same.

The preliminary investigation of TSAAG based on contact ratio reveals the possibility of achieving high contact ratio (HCR) TSAAG with positive values of tooth alterations $(\alpha>1)$ and low contact ratio (LCR) TSAAG, with negative values of tooth alterations $(\alpha<1)$ and all gear pairs running on the same center distance. Experimental results by Kasuba [16] and Kahraman-Blankenship [17] have established that the dynamic loads decrease with increasing contact ratio in spur gearing. Even though TSAAG with HCR > 2 opens up the possibility of transmitting more power at low decibels without resorting to higher strength materials, it is important to interpret this result in the light of safe load transmission capability.

The plot of safe load-bearing capability $F$ vs $\alpha$ (refer to Fig. 10) shows an increase in load-bearing capability on either side of unity but the interpretation varies. The increase in load-bearing ability for LCR TSAAG with $\alpha<1$ is attributed to the increased tooth thickness and pressure angles. However, for HCR TSAAG, the increase in load-bearing ability comes due to the contact ratio rising above 2 as seen in the case of $\alpha=1.01852$. Further Increasing the value of $\alpha$, reduces load-bearing ability due to the reduction in tooth thickness. The product of dynamic load factor and location factor for bending $x_{d l} \times k_{t}$, referring to Figs. 11 (a)-(f), is the sole dimensionless parameter that decides the safe load-bearing ability of any gear. Normal contact ratio standard tooth sum (NCR STS) asymmetric gears and LCR TSAAG show one region of single tooth contact and two regions of double tooth contact. HCR TSAAG shows three regions of triple teeth contact and two regions of double tooth contact, indicating an improvement in the load-bearing capacity. The maximum value of $x_{d l} \times k_{t}$ in either of the gears decides the safe load transmission capability of any gear drive. Dynamic load factor is speed-dependent and location factor for bending is geometry dependent.

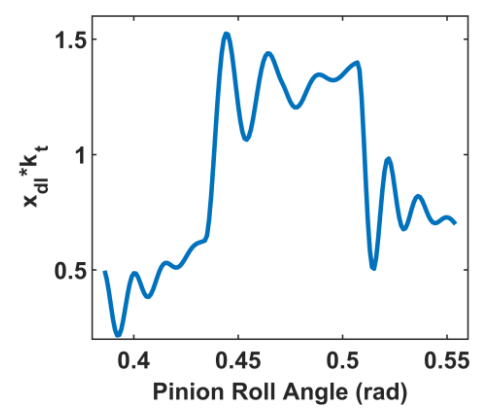

(a) $\alpha=0.96296, \kappa=0.5, \beta=1$

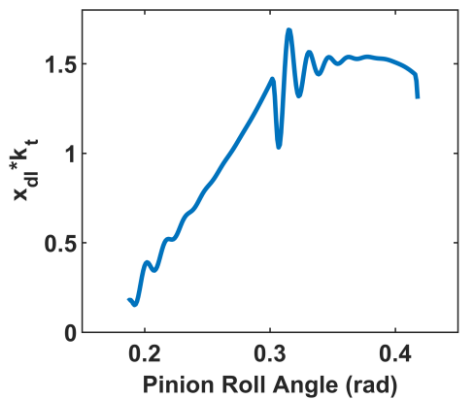

(d) $\alpha=1.01852, \kappa=0.5, \beta=1$

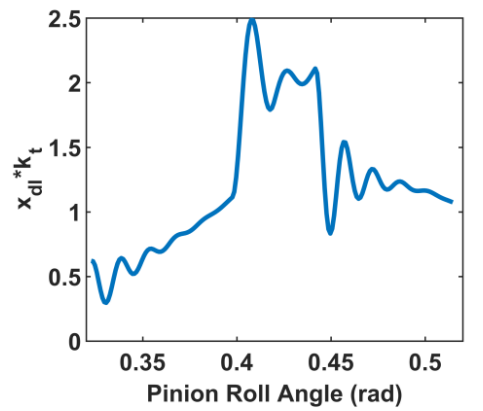

(b) $\alpha=0.98148, \kappa=0.5, \beta=1$

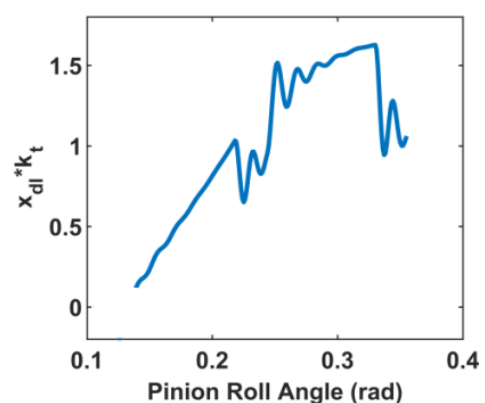

(e) $\alpha=1.3703, \kappa=0.5, \beta=1$

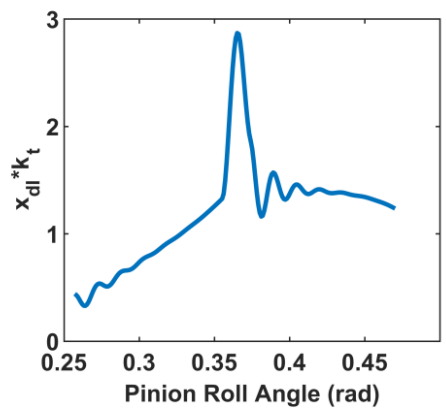

(c) $\alpha=1, \kappa=0.5, \beta=1$

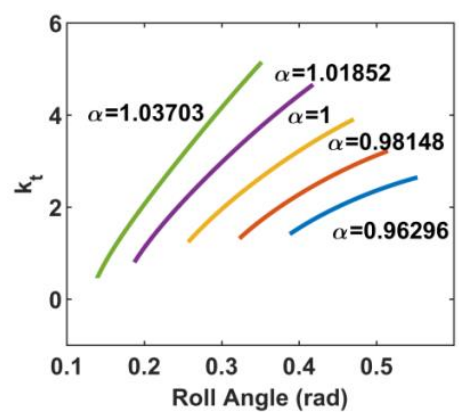

(f) Location factors $k_{t}$ for bending

Fig. $11 x_{d l} \times k_{t}$ for $0.96296 \leq \alpha \leq 1.3703$ 
The single tooth contact region is the largest for $\alpha=0.96296$ and starts reducing as alpha approaches unity. The dynamic load dominates the magnitude of $x_{d l} \times k_{t}$ which is evident by observing the slope of the plots for $\alpha<1$. At any given speed, dynamic load in a gear system decreases with an increasing contact ratio. This is because of the narrow single or triple contact zone, which passes quickly, leaving no time for the system to respond. Hence for HCR TSAAG the value of $k_{t}$ dominates the magnitude of $x_{d l} \times k_{t}$. The plot of TSAAG with $\alpha=1.01852$ yields the lowest of the maximum magnitude of $x_{d l} \times k_{t}$ among TSAAG, which gives a clear impression of having the largest load-bearing ability. Also, HCR TSAAG is expected to have lower decibel levels due to reduced dynamic loads.

While it looks very encouraging to know that it is possible to have improved load transmission capability with HCR TSAAG, it is also important to evaluate its surface durability. The product of dynamic load factor and specific sliding $x_{d l} \times V_{s s}$ is the sole dimensionless parameter that decides the wear accumulation, refering to Eq. (32). The gear pairs are subjected to a load value to sustain $10^{6}$ bending cycles. At $\kappa=0.5$, TSAAG as well as NCR asymmetric gears, irrespective of the value of $\alpha$, attain equal approach-recess action. The plots of $x_{d l} \times V_{s s}$ for TSAAG operating under $\kappa=0.5$ with different $\alpha$ values, accumulated over $10^{5}$ cycles are shown in Figs. 12(a)-(f). For TSAAG with $\alpha<1$ the slope of the curve is steeper in the region of single tooth contact owing to comparatively larger load and it reduces in the two teeth contact region. The region on either side of the pitch point covering a certain roll angle indicates a larger region of single tooth contact or lower contact ratio. The gears experience larger dynamic loads during the transition from single tooth to double tooth contact at lower speeds. The specific sliding in Fig. 12(f) shows significant improvement for $\alpha<1$ in the addendum and dedendum regions of both pinion and the gear. The improvement in specific sliding offsets the higher dynamic loads, keeping the accumulation of $x_{d l} \times V_{s S}$ minimal up to $10^{5}$ cycles. A comparison with asymmetric NCR gear indicates higher service life of TSAAG LCR with higher decibel levels at lower speeds due to reduced contact ratio.

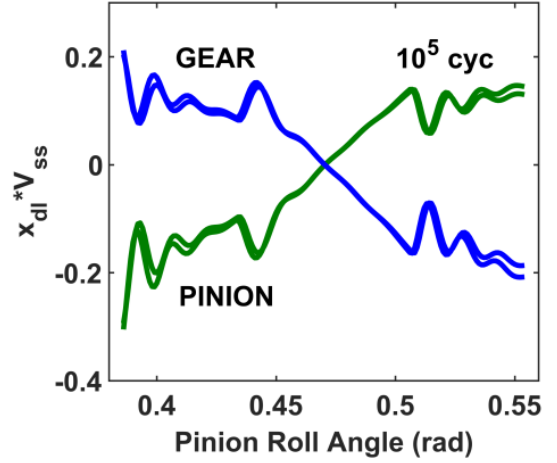

(a) $\alpha=0.96296, \kappa=0.5, \beta=1$

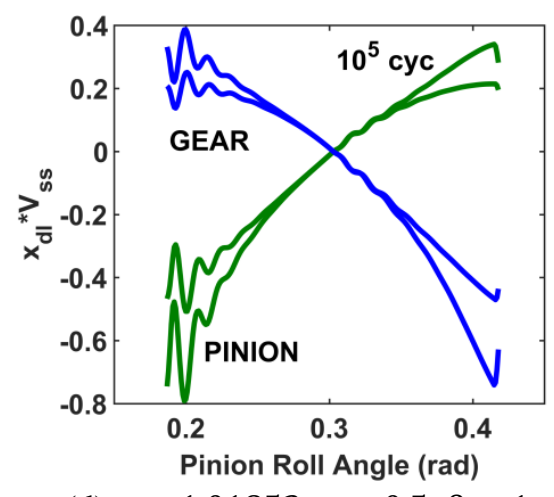

(d) $\alpha=1.01852, \kappa=0.5, \beta=1$

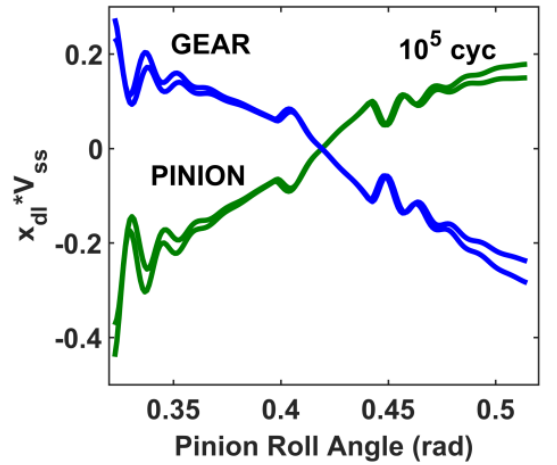

(b) $\alpha=0.98148, \kappa=0.5, \beta=1$

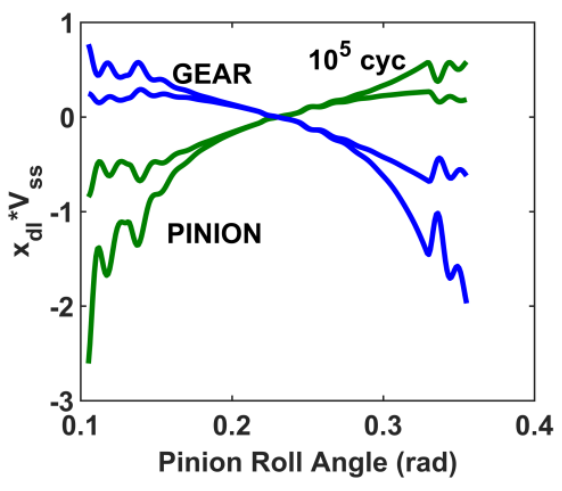

(e) $\alpha=1.03703, \kappa=0.5, \beta=1$

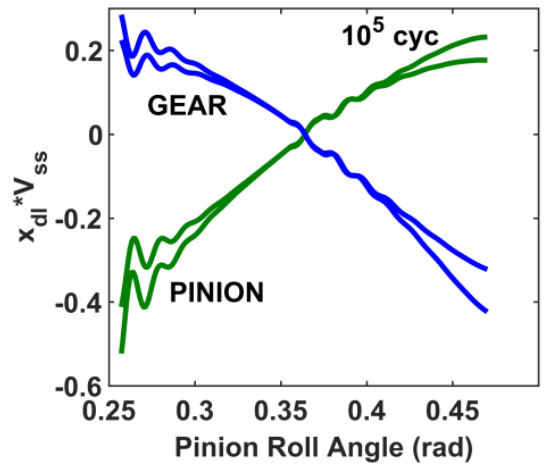

(c) $\alpha=1, \kappa=0.5, \beta=1$

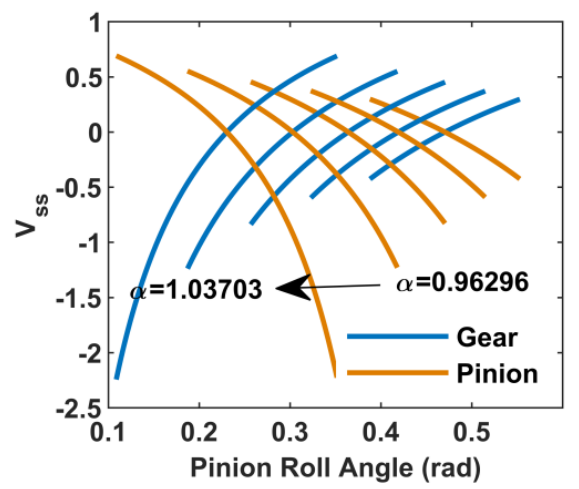

(f) Specific sliding $V_{s S}$

Fig. $12 x_{d l} \times V_{s s}$ for $0.96296 \leq \alpha \leq 1.3703$ (continued)

The accumulation of $x_{d l} \times V_{S S}$ for TSAAG HCR as the value of $\alpha$ approaches 1.03703 is significant over $10^{5}$ cycles at the region near the root and addendum circles, where the slope of specific sliding changes drastically. Profile error introduced due to wear is accumulative over cycles of usage. The dynamic loads are lower as a result of a narrow region of three teeth contact 
which passes quickly. The dynamic load factor increases with the accumulation of profile error. Specific sliding considered constant for every cycle acts as an amplifier to the iterating dynamic load factors. Hence the accumulation of $x_{d l} \times V_{S S}$ becomes large especially at locations having higher specific sliding. While the dynamic load in STS asymmetric gears and TSAAG LCR with $\alpha<1$ does not grow as much owing to lower wear rates, TSAAG HCR gears with $\alpha>1$, start with lower dynamic loads and build up to higher-value during the course of its life. These gears are expected to run with decibel levels lower than the STS asymmetric gears for an initial period, but the vibrations and noise levels increase over their service life.

\section{Conclusions}

Altering the tooth sum of a reference NCR asymmetric gear pair offers a family of TSAAG gears for the same center distance with improved performance characteristics. Tooth sum is a macroscopic parameter that alters pressure angle and tooth thickness offering the flexibility of accommodating various tooth sums on the same center distance. Increased tooth-sum coupled with reduced pressure angle and tooth topping results in HCR TSAAG and decreasing tooth sum results in LCR TSAAG. Both LCR and HCR TSAAG have improved load-bearing ability and mesh stiffness. The effects of tooth-sum alteration on surface wear are influenced by meshing parameters such as dynamic load factors, specific sliding, and material properties. Wear rate increases for TSAAG, as $\alpha$ moves away from unity either towards lesser or greater values within the permissible limits. The specific sliding and dynamic load factors play a significant role in determining the overall dynamic load, surface wear build-up, and service life. The rate of change of slope of the specific sliding curve is a dominant factor influencing the wear rate and tooth life. HCR TSAAG offers up to 50\% more load-bearing ability than NCR asymmetric gears, but higher wear rates increase dynamic loads which may reduce service life. LCR TSAAG has up to 13\% more load-bearing ability with better surface durability but suffers loss in contact ratio which may cause noisy operation. In conclusion, tooth sum modified asymmetric gear geometry (TSAAG) is an effective design technique to introduce geometrical changes for design benefits while maintaining the center distance constant as they offer design benefits that are not achievable by NCR asymmetric gear design.

\section{Nomenclature}

\begin{tabular}{|c|c|c|c|}
\hline$\alpha$ & Tooth-sum alteration factor & $\mathrm{B}$ & Design Backlash \\
\hline$\beta$ & Center distance alteration factor & $c$ & Center distance \\
\hline$\ddot{x}_{r}$ & Relative acceleration at mesh interface & $c_{e q}$ & Equivalent Damping coefficient \\
\hline$\dot{x}_{r}$ & Relative velocity at mesh interface & $E$ & Modulus of elasticity \\
\hline$\kappa$ & Profile shift factor & $F$ & Total static load per unit face width \\
\hline$\lambda$ & Mesh stiffness per unit face width & $F_{\lambda}$ & Spring force \\
\hline$\mu$ & Coefficient of friction & $F_{c}$ & Damping force \\
\hline$\phi$ & Pressure angle & $f_{d x}$ & Location-based dynamic load per unit face width \\
\hline$\rho$ & Radius of curvature & $h$ & Accumulated wear \\
\hline$\sigma_{f}$ & Allowable bending fatigue strength & $k_{e q}$ & Equivalent Mesh stiffness \\
\hline$\theta_{r a}$ & Roll angle of addendum radius & $k_{t}$ & Tooth geometric factor for bending \\
\hline$\theta_{r l}$ & Roll angle of radius of limiting circle & $k_{w}$ & Wear Coefficient \\
\hline$\xi$ & Damping factor & $m$ & Module \\
\hline$\varepsilon$ & Tooth profile error & $r_{b}$ & Radius of Base circle \\
\hline$\sigma_{f c}$ & Contact pressure & $x$ & Profile shift coefficient \\
\hline$r_{l}$ & Radius of limit circle & $x_{s l}$ & Static load factor \\
\hline$r_{f}$ & Radius of the fillet circle & $x_{d l}$ & Dynamic load factor \\
\hline$r_{x}$ & Radius of any point on the profile & $x_{r}$ & Relative displacement at mesh interface \\
\hline$T$ & Total static torque per unit face width & $Y$ & Tooth topping coefficient \\
\hline$v_{r}$ & Rolling velocity & $z$ & Number of teeth \\
\hline$V_{s s}$ & Specific sliding & $Z_{s}$ & Tooth-sum of gear pair \\
\hline$X_{s}$ & Total profile shift coefficient & & \\
\hline & & & \\
\hline
\end{tabular}




\begin{tabular}{|c|c|c|c|}
\hline \multicolumn{2}{|l|}{ Subscripts } & \multicolumn{2}{l|}{ Superscripts } \\
\hline 1,2 & Driver and driven gears & $a$ & Altered \\
\hline$s$ & Standard & $r$ & Reference \\
\hline$w$ & Working & $d$ & Drive side \\
\hline$x$ & Any location along the pressure line & $c$ & Coast side \\
\hline$p$ & Pitch circle & \multicolumn{2}{|c|}{} \\
\hline Abbreviations & NCR & Normal contact ratio \\
\hline TSAAG & Tooth sum altered asymmetric gear & rpm & Revolutions per minute \\
\hline HCR & High contact ratio & STS & Standard tooth-sum \\
\hline LCR & Low contact ratio &
\end{tabular}

\section{Conflicts of Interest}

The authors declare no conflict of interest.

\section{References}

[1] A. Kapelevich, "Geometry and Design of Involute Spur Gears with Asymmetric Teeth," Mechanism and Machine Theory, vol. 35, no. 1, pp. 117-130, January 2000.

[2] F. L. Litvin, Q. Lian, and A. L. Kapelevich, "Asymmetric Modified Spur Gear Drives: Reduction of Noise, Localization of Contact, Simulation of Meshing and Stress Analysis," Computer Methods in Applied Mechanics \& Engineering, vol. 188, no. 1-3, pp. 363-390, July 2000.

[3] F. Karpat, S. Ekwaro-Osire, K. Cavdar, and F. C. Babalik, "Dynamic Analysis of Involute Spur Gears with Asymmetric Teeth,” International Journal of Mechanical Sciences, vol. 50, no. 12, pp. 1598-1610, December 2008.

[4] F. Karpat and S. Ekwaro-Osire, "Influence of Tip Relief Modification on the Wear of Spur Gears With Asymmetric Teeth," Tribology Transactions, vol. 51, no. 5, pp. 581-588, September 2008.

[5] R. Prabhu Sekar, "Performance Enhancement of Spur Gear Formed Through Asymmetric Tooth," Proceedings of the Institution of Mechanical Engineers, Part J: Journal of Engineering Tribology, vol. 233, no. 9, pp. 1361-1378, September 2019.

[6] R. Prabhu Sekar and G. Muthuveerappan, "Estimation of Tooth Form Factor for Normal Contact Ratio Asymmetric Spur Gear Tooth,” Mechanism \& Machine Theory, vol. 90, pp. 187-218, August 2015.

[7] J. Gonsalvis and G. Rayudu, "Varying the Tooth-Sum of a Gear Pair Operating on a Specified Center Distance-Its Effects on Contact Ratio and Gear Ratio," Proceedings of the National Conference of Mechanisms and Machines, pp. 139-146, 1990.

[8] A. A. Dsa and J. Gonsalvis, "Investigation on Load Distribution Based on Combined Stiffness in Altered Tooth-Sum Gears," International Conference on Mechanical Power Transmission, pp. 2-7, July 2019.

[9] J. Gonsalvis and H. Sachidananda, "Altered Tooth-Sum Gearing for High Contact Ratio," International Journal of Engineering Research and Applications, vol. 1, no. 3, pp. 1234-1241, Octorber 2011.

[10] K. Ichimaru and H. Fujio, "Dynamic Behavior of Heavy-Loaded Spur Gears," Journal of Engineering for Industry, pp. 373-381, 1974.

[11] S. M. Arikan, "Dynamic Load and Contact Stress Analysis of Spur Gears," American Society of Mechanical Engineers, Design Engineering Division (Publication) DE, vol. 32, no. pt 1, pp. 85-91, 1991.

[12] G. Maitra, Handbook of Gear Design, New Delhi: Tata McGraw Hill, 1994.

[13] J. R. Colbourne, The Geometry of Involute Gears, New York: Springer Verlag New York, 1987.

[14] J. F. Archard, "Contact and Rubbing of Flat Surfaces," Journal of Applied Physics, vol. 24, no. 8, pp. 981-988, August 1953.

[15] A. Flodin and S. Andersson, "Simulation of Mild Wear in Spur Gears," Wear, vol. 207, no. 1-2, pp. 16-23, June 1997.

[16] R. Kasuba, "Dynamic Loads in Normal and High Contact Ratio Spur Gearing," International Symposium on Gearing and Power Transmissions, 1981, pp. 49-54.

[17] A. Kahraman and G. W. Blankenship, "Effect of Involute Contact Ratio on Spur Gear Dynamics," Journal of Mechanical Design, vol. 121, no. 1, pp. 112-118, March 1999.

Copyright $\odot$ by the authors. Licensee TAETI, Taiwan. This article is an open access article distributed under the terms and conditions of the Creative Commons Attribution (CC BY-NC) license

(https://creativecommons.org/licenses/by-nc/4.0/). 